\title{
APPLICATIONS OF EXTREME POINT THEORY TO CLASSES OF MULTIVALENT FUNCTIONS
}

\author{
BY \\ DAVID J. HALLENBECK AND ALBERT E. LIVINGSTON ( ${ }^{1}$ )
}

\begin{abstract}
Extreme points of the closed convex hulls of several classes of multivalent functions are determined. These are then used to determine the precise bounds on the coefficients of a function majorized by or subordinate to a function in any of the classes. $L^{q}$ means are also discussed and subordination theorems are considered. The classes we consider are generalizations of the univalent starlike, convex and close-to-convex functions in addition to others.
\end{abstract}

I. Introduction. Recently there has been considerable interest in determining the extreme points of the closed convex hulls of certain classes of univalent functions and using these determinations to solve certain extremal problems [3], [4], [10], [18]. It is the purpose of this paper to extend some of these considerations to certain classes of multivalent functions. Let $S(p, q), p$ and $q$ integers, $1 \leqslant q \leqslant p$, be the class of functions $f(z)$ analytic in $\Delta=\{z:|z|$ $<1\}$ with power series expansion $f(z)=z^{q}+\Sigma_{n=q+1}^{\infty} a_{n} z^{n}, z \in \Delta$, and for which there exists a $\rho=\rho(f)$ such that

$$
\operatorname{Re}\left(z f^{\prime}(z) / f(z)\right)>0
$$

and

$$
\int_{0}^{2 \pi} \operatorname{Re} \frac{z f^{\prime}(z)}{f(z)} d \theta=2 p \pi \quad \text { for } z=r e^{i \theta}, \rho<r<1 .
$$

Functions in $S(p, q)$ are $p$-valent and are referred to as multivalent starlike functions [6]. We let $S^{*}(p, q)$ be the subclass of $S(p, q)$ of functions which are regular on $|z|=1$ and satisfy (1.1) and (1.2) on $|z|=1$. It is known [6] that if $f(z)$ is in $S(p, q)$, then it has exactly $p$ zeros in $\Delta$ and, moreover, if $f(z)$ has $q$ zeros at the origin and $(p-q)$ zeros at $\alpha_{1}, \alpha_{2}, \ldots, \alpha_{p-q}, 0<\left|\alpha_{i}\right|<1$, then

Presented to the Society, August 24, 1974; received by the editors September 23, 1974 and, in revised form, June 9, 1975.

AMS (MOS) subject classifications (1970). Primary 30A32, 30A34, 30A40.

Key words and phrases. Extreme point, closed convex hull, multivalent function, multivalent starlike function, multivalent convex function, multivalent close-to-convex function, majorization, subordination, $L^{q}$ means.

( ${ }^{1}$ ) The research of the first author was partially supported by a University of Delaware Faculty Research Summer Grant for the period June, 1974, to September, 1974. 


$$
f(z)=\prod_{k=1}^{p-q} \frac{\left(1-z / \alpha_{k}\right)\left(1-\overline{\alpha_{k} z}\right)}{z^{p-q}}(g(z))^{p}
$$

where $g(z)$ is in $S(1,1)$, the class of normalized univalent starlike functions. If we let $F(p, q)$ denote the class of functions which have the representation (1.3) then $S(p, q) \subset F(p, q)$ and the containment is proper [1], [6] .

A function $f(z)=z^{q}+\sum_{n=q+1}^{\infty} a_{n} z^{n}, z \in \Delta$, is said to be in $C(p, q)$ if and only if $z f^{\prime}(z) / q$ is in $S(p, q)$. Functions in $C(p, q)$ are called multivalent convex functions. It should be noted [6] that $C(p, q) \not S S(p, q)$ if $q \neq p$, but $C(p, p) \subset S(p, p)$. As we have already pointed out, a function in $S(p, p)$ is the $p$ th power of a univalent starlike function; however, it is easily seen that a function in $C(p, p)$ is not necessarily the $p$ th power of a univalent convex function. Suppose $f(z)=g(z)^{p}$ where $g(z)$ is in $C(1.1)$. Then from the well-known result $\operatorname{Re}\left(z g^{\prime}(z) / g(z)\right)>1 / 2 \quad[20]$, it would follow that $\operatorname{Re}\left(1+z f^{\prime \prime}(z) / f^{\prime}(z)\right)>$ $(p-1) / 2$. However, $f(z)=p \int_{0}^{z} \tau^{p-1} /(1-\tau)^{2 p} d \tau$ is in $C(p, p)$ and $1+$ $z f^{\prime \prime}(z) / f^{\prime}(z)=p(1+z) /(1-z)$ maps $\Delta$ onto the right half plane.

Analogues of the classical results of Strohhäcker [23] and Marx [20] that $\operatorname{Re} z f^{\prime}(z) / f(z)>1 / 2$ and $\operatorname{Re} f(z) / z>1 / 2$ for a function in $C(1,1)$ do not hold in $C(p, p)$. That is, there is no constant $A>0$ such that $\operatorname{Re} z f^{\prime}(z) / f(z)>A, z \in$ $\Delta$, for all $f(z)$ in $C(p, p)$, and the quantity $\operatorname{Re} f(z) / z^{p}$ can be negative. [Note that the inequality $\operatorname{Re} z f^{\prime}(z) / f(z)>0, z \in \Delta$, follows by virtue of the fact that $C(p, p) \subset S(p, p)$.] To see this we consider the function

$$
F(z)=2 \int_{0}^{z} \frac{\tau}{(1-\tau)^{4}} d \tau=\frac{3 z^{2}-z^{3}}{3(1-z)^{3}}
$$

which is in $C(2,2)$. A straightforward computation shows that for $z=e^{i \theta}$ and $x=\cos \theta$,

$$
\operatorname{Re} 3 F(z) / z^{2}=-(1-x)^{2}(1+3 x) / 2(1-x)^{3}<0
$$

for $-1 / 3<x<1$, and if $z=e^{i \theta}, \theta \neq 0$,

$$
\operatorname{Re} z F^{\prime}(z) / F(z)=3\left|1-e^{i \theta}\right|^{2} /\left|3-e^{i \theta}\right|^{2}
$$

which approaches 0 as $\theta$ approaches zero.

A function $f(z)$ is said to be subordinate to $F(z)$ in $\Delta$ if $f(z)$ and $F(z)$ are analytic in $\Delta, f(0)=F(0)$, and there exists $w(z)$ analytic in $\Delta$ with $w(0)=0$ and $|w(z)|<1, z \in \Delta$, such that $f(z)=F(w(z))$. In the case that $F$ is univalent, then $f$ is subordinate to $F$ is equivalent to $f(0)=F(0)$ and $f(\Delta) \subset F(\Delta)$. We will use the notation $f<F$ to indicate that $f$ is subordinate to $F$. The results of Strohhäcker and Marx concerning $C(1,1)$ can be viewed as saying that $z f^{\prime}(z) / f(z) \prec z F^{\prime}(z) / F(z)$ and $f(z) / z \prec F(z) / z$ where $F(z)=z /(1-z)$. It seems 
reasonable to conjecture that if $f(z)$ is in $C(p, p)$, then $z f^{\prime}(z) / f(z)<z F^{\prime}(z) / F(z)$ and $f(z) / z^{p} \prec F(z) / z^{p}$ where $F(z)=p \int_{0}^{z} \tau^{p-1} /(1-\tau)^{2 p} d \tau$. In $\S \mathrm{V}$ we strengthen these conjectures by showing that they are true for the cases $p=2$ and 3 .

We define the class $K(p, q)$ [16] to be the class of functions $f(z)=z^{q}+$ $\sum_{n=q+1}^{\infty} a_{n} z^{n}, z \in \Delta$, for which there exist $g(z)$ in some $S(p, t)$ and an $\alpha, 0 \leqslant \alpha$ $\leqslant 2 \pi$, and a $\rho, 0<\rho<1$, such that

$$
\operatorname{Re}\left(e^{i \alpha} z f^{\prime}(z) / g(z)>0 \text { for } \rho<|z|<1 .\right.
$$

Functions in $K(p, q)$ are at most $p$-valent and their derivatives have exactly $(p-1)$ zeros in $\Delta[16]$. Functions in $K(p, q)$ are called multivalent close-toconvex functions and $K(1,1)$ is the class of univalent close-to-convex functions defined by Kaplan [14]. It is obvious that $S(p, q) \subset K(p, q)$. We let $K^{*}(p, q)$ be the subclass of functions $f(z)$ in $K(p, q)$ which are analytic on $|z|=1$ and for which there exist $g(z)$ in $S^{*}(p, t)$ and an $\alpha, 0 \leqslant \alpha \leqslant 2 \pi$, such that (1.4) holds on $|z|=1$. In what follows we will have need of the following lemma about $K(p, q)$. The lemma is implicitly contained in [16], but we include a proof for the sake of completeness.

Lemma (1.1). Let $f(z)$ be in $K(p, p)$; then there exist $g(z)$ in $S(p, p)$ and an $\alpha, 0 \leqslant \alpha \leqslant 2 \pi$, such that

$$
\operatorname{Re}\left(e^{i \alpha} z f^{\prime}(z) / g(z)\right)>0 \text { for } z \text { in } \Delta .
$$

Proof. If $f(z)$ is in $K^{*}(p, p)$, then according to [16, Lemma 3] there exist $g(z)$ in $S^{*}(p, p)$ and an $\alpha, 0 \leqslant \alpha \leqslant 2 \pi$, such that $\operatorname{Re}\left(e^{i \alpha} z f^{\prime}(z) / g(z)\right)>0$ on $|z|=1$. Since $z f^{\prime}(z) / g(z)$ is analytic in $|z| \leqslant 1$, we have $\operatorname{Re}\left(e^{i \alpha} z f^{\prime}(z) / g(z)\right)>0$ for $|z| \leqslant 1$. If $f(z)$ is in $K(p, p)$ then there exists $\rho, 0<\rho<1$, such that $f_{r}(z)=r^{-p} f(r z)$ is in $K^{*}(p, p)$ if $\rho<r<1$. Thus for each $r, \rho<r<1$, there exists $g_{r}(z)$ in $S^{*}(p, p)$ and $\alpha_{r}, 0 \leqslant \alpha_{r} \leqslant 2 \pi$ such that $\operatorname{Re}\left(e^{i \alpha_{r}} f_{r}^{\prime}(z) / g_{r}(z)\right)>0$ for $z$ in $\Delta$. Since $S(p, p)$ is normal and compact we can choose a sequence $r_{k}$ tending to 1 such that $g_{r_{k}}(z) \rightarrow g(z)$ in $S(p, p)$. [Note that the family $S(p, q)$, $q \neq p$, is not compact [1].] We may assume that $\alpha_{r_{k}} \rightarrow \alpha$. A passage to the limit then gives $\operatorname{Re}\left(e^{i \alpha} z f^{\prime}(z) / g(z)\right) \geqslant 0$. We note that equality cannot occur and so this proves the lemma.

We will also consider a class of functions $T(p)$ studied by Goodman and Robertson [7]. Let $f(z)$ be analytic in $\Delta$; then $\operatorname{Im} f(z)$ is said to change sign $2 p$ times on $|z|=r$ if there are exactly $2 p$ points $z=r e^{i \theta} j, j=1,2, \ldots, 2 p$, such that $\operatorname{Im} f\left(r e^{i \theta} j\right)=0$ for $j=1,2, \ldots, 2 p$, and for each $j$ there exists an $\epsilon>0$ such that

$$
\operatorname{Im} f\left(r e^{i\left(\theta_{j}+\delta\right)}\right) \operatorname{Im} f\left(r e^{i\left(\theta_{j}-\delta\right)}\right)<0 \text { for } 0<\delta<\epsilon .
$$

A function $f(z)=z^{p}+\sum_{n=1}^{\infty} a_{n} z^{n}$ is said to be in $T(p)$ if $a_{n}$ is real for all $n$ 
and there exists $\rho, 0<\rho<1$, such that for each $r, \rho<r<1$, Im $f(z)$ changes sign $2 p$ times on $|z|=r$. We say $f(z)=z^{p}+\Sigma_{n=p+1}^{\infty} a_{n} z^{n}$ is in $T^{*}(p)$ if the $a_{n}$ are real, $f(z)$ is analytic for $|z| \leqslant 1$ and $\operatorname{Im} f(z)$ changes sign $2 p$ times on $|z|=1$. It is easily seen that those functions in $S(p, p)$ with real coefficients in their power series are contained in $T(p)$ [7].

If $B$ is a class of functions analytic in $\Delta$, we denote the closed convex hull of $B$ by $H B$ and the set of extreme points of the closed convex hull of $B$ by $E H B$. We determine $H S(p, p), H C(p, p), H K(p, p), H T(p)$ and also $E H S(p, p)$, $\operatorname{EHC}(p, p), \operatorname{EHK}(p, p)$ and $\operatorname{EHT}(p)$. We also treat completely the subclasses of each of the above classes determined by those functions in each class which have real coefficients.

The knowledge of extreme points then allows us to solve certain extremal problems over various classes. In particular, we find the sharp bounds on the coefficients of any function $f(z)$ such that $f(z)<F(z)$ where $F(z)$ is in one of the classes $S(p, p), C(p, p), K(p, p)$ or $T(p)$.

If $f(z)$ and $g(z)$ are analytic in $\Delta$, then $f(z)$ is majorized by $g(z)$ if $|f(z)| \leqslant$ $|g(z)|$ for $z$ in $\Delta$. This implies that there exists a function $\phi(z)$ analytic in $\Delta$ so that $|\phi(z)| \leqslant 1$ and $f(z)=\phi(z) g(z)$ [17]. In §III we use the knowledge of extreme points to determine precise coefficient bounds on any function $f(z)$ majorized by a function $g(z)$ in $S(p, p), C(p, p), K(p, p)$ or $T(p)$.

In $\S I V$ we consider the quantities $\int_{0}^{2 \pi} \mid f\left(r e^{i \theta}\right)^{q} d \theta, 0<r<1, q \geqslant 1$, where $f$ ranges over various classes of functions. Let $K(z)=z^{p} /(1-z)^{2 p}$; then we prove that

$$
\int_{0}^{2 \pi}\left|f^{(n)}\left(r e^{i \theta}\right)^{\mid q} d \theta \leqslant \int_{0}^{2 \pi}\right| K^{(n)}\left(r e^{i \theta}\right)^{q} d \theta
$$

where $f^{(n)}$ denotes the $n$th derivative of $f, n=0,1, \ldots, q \geqslant 1$, and $f$ is in $S(p, p)$ or $T(p)$. A similar result is proven for $C(p, p)$ where $K(z)$ is replaced by $F(z)=p \int_{0}^{z} \tau^{p-1} /(1-\tau)^{2 p} d \tau$. Furthermore, if $f(z)$ is in $K(p, p)$, then we prove that

$$
\begin{gathered}
\int_{0}^{2 \pi}\left|f\left(r e^{i \theta}\right)\right|^{q} d \theta \leqslant \int_{0}^{2 \pi} \mid K\left(r e^{i \theta}\right)^{q} d \theta, \quad q=1,2,3, \ldots ; \\
\int_{0}^{2 \pi}\left|f^{(1)}\left(r e^{i \theta}\right)\right|^{q} d \theta \leqslant \int_{0}^{2 \pi}\left|K^{(1)}\left(r e^{i \theta}\right)\right|^{q} d \theta, \quad q \geqslant 1,
\end{gathered}
$$

and

$$
\int_{0}^{2 \pi}\left|f^{(n)}\left(r e^{i \theta}\right)\right|^{q} d \theta \leqslant \int_{0}^{2 \pi}\left|K^{(n)}\left(r e^{i \theta}\right)\right|^{q} d \theta, \quad n \geqslant 2, q=2,4,6, \ldots .
$$

Finally, in the sequel we will use the notation $f(z)<<g(z)$ to indicate 
that $f(z)=\Sigma_{n=0}^{\infty} a_{n} z^{n}, g(z)=\sum_{n=0}^{\infty} b_{n} z^{n}, z \in \Delta, b_{n} \geqslant 0$ for all $n$ and $\left|a_{n}\right| \leqslant$ $b_{n}$ for all $n$. Also if $A$ denotes a class of functions analytic in $\Delta$, then $A_{R}$ will denote the subclass of functions in $A$ whose power series expansions in $\Delta$ have real coefficients.

II. The convex hulls and extreme points of $C(p, p), S(p, p), K(p, p), T(p)$, $C(p, p)_{R}, S(p, p)_{R}$, and $K(p, p)_{R}$. We let

$$
F_{p}(g)=\left\{\int_{X} g^{p}(z, x) d \mu(x): \mu \in P\right\}
$$

where $p>0$ and $P$ is the set of probability measures on $X=\{x:|x|=1\}$. If $g(z, x)=1 /(1-x z)$, then in [2] the following lemma is proven:

LeMMA (2.1). $F_{p} \circ F_{q} \subset F_{p+q}$.

If $X=\{x:|x|=1$ and $\operatorname{Im} x \geqslant 0\}$ and $g(z, x)=1 /(1-x z)(1-\bar{x} z)$, then in [9] the following lemma is proven:

Lemma (2.2). $\quad F_{p} \circ F_{q} \subset F_{p+q}$.

THEOREM (2.1). Let $X$ be the unit circle, $P$ the set of probability measures on $X$, and $F$ the set of functions $f_{\mu}$ on $\Delta$ defined by

$$
f_{\mu}(z)=\int_{X} \frac{z^{p}}{(1-x z)^{2 p}} d \mu(x), \quad \mu \in P .
$$

Then $F=H S(p, p)$, the map $\mu \rightarrow f_{\mu}$ is one-to-one, and the extreme points of $H S(p, p)$ are precisely the kernel functions in equation (2.1.1).

Proof. The kernel functions are in $S(p, p)$ for each $|x|=1$ and, hence, $F \subset H S(p, p)$. Let $f(z) \in S(p, p)$. We know that $f(z)=g(z)^{p}$ where $g(z)$ is starlike univalent. Hence by a result in [3] we have

$$
f(z)=\left[\int_{X} \frac{z}{(1-x z)^{2}} d \mu(x)\right]^{p} \text {. }
$$

By Lemma (2.1) we conclude that $f(z) \in F$. Hence by Theorem (1) in [3] we have $H S(p, p)=F$. If $f_{\mu_{1}}=f_{\mu_{2}}$, then it is easily shown that $\int_{X} x^{n} d \mu_{1}=$ $\int_{X} x^{n} d \mu_{2}$ for $n=1,2, \ldots$ and, consequently, $\mu_{1}=\mu_{2}$. The remark about the extreme points now follows by the result quoted above [3].

REMARKS. We note at this time that the extreme points of the closed convex hull of the subclass of $F(p, q)$ of functions with fixed zeros $\alpha_{1}, \alpha_{2}, \ldots$, $\alpha_{(p-q)},\left|\alpha_{i}\right|<1,0 \leqslant q \leqslant p$, are exactly the functions

$$
\prod_{K=1}^{p-q} \frac{\left(1-z / \alpha_{k}\right)\left(1-\bar{\alpha}_{k} z\right)}{z} \frac{z^{p}}{(1-x z)^{2 p}} \text { where }|x|=1 .
$$

Similar results can be obtained for $C(p, q)$ and $K(p, q)$ by keeping the zeros of 
the derivative fixed. We do not include these results since a determination of the extreme points in these cases does not allow us to solve any interesting extremal problems except for the case of majorization for $S(p, q)$.

TheOREM (2.2). Let $X$ be the unit circle, $P$ the set of probability measures on $X$, and $F$ the set of functions $f_{\mu}$ on $\Delta$ defined by

$$
f_{\mu}(z)=\int_{X}\left[\int_{0}^{z} \frac{p \tau^{p-1}}{(1-x \tau)^{2 p}} d \tau\right] d \mu(x)
$$

Then $F=H C(p, p)$, the map $\mu \rightarrow f_{\mu}$ is one-to-one and the extreme points of $H C(p, p)$ are precisely the kernel functions in equation (2.2.1).

Proof. Let $(L f)(z)=p \int_{0}^{z} f(\tau) / \tau d \tau$. We know that $L(S(p, p))=C(p, p)$. Since the operator is a homeomorphism the theorem follows immediately from the previous theorem.

REMARKs. (1) We are also able to treat completely the case of $n$-fold symmetric functions in $S(p, p)$ and $C(p, p)$. A function $f(z)=z^{p}+A_{1} z^{p+1}$ $+\cdots$ is said to be $n$-fold symmetric if it has the form $\Sigma_{k=0}^{\infty} A_{k} z^{p+k n}$. If $f(z)$ is in $S(p, p)$ and is $n$-fold symmetric, it is easy to see that $f(z)=g(z)^{p}$ where $g(z)$ is $n$-fold symmetric in addition to being starlike univalent. Results analogous to Theorems 2.1 and 2.2 can now be proven for these $n$-fold symmetric functions by the same type of arguments used above by appealing to Theorem (3) in [4] We do not include these results explicitly since we do not use them in the applications.

(2) We note at this time that it is known [6] that

$$
F(z)=\int_{0}^{z} \frac{p \tau^{p-1}}{(1-\tau)^{2 p}} d \tau=\frac{\sum_{n=1}^{2 p-1}(-1)^{n+p} p !\left({ }^{2 p-1}\right) z^{n}}{(2 p-1) \ldots(p)(1-z)^{2 p-1}}
$$

This claim can be verified by differentiation.

TheOREM (2.3). Let $X=\{x:|x|=1, \operatorname{Im} x \geqslant 0\}, P$ be the set of probability measures on $X$, and $F$ the set of functions $f_{\mu}$ on $\Delta$ defined by

$$
f_{\mu}(z)=\int_{X} \frac{z^{p}}{(1-x z)^{p}(1-\bar{x} z)^{p}} d \mu(x), \quad \mu \in P .
$$

Then $F=H S(p, p)_{R}=T(p)$, the map $\mu \rightarrow f_{\mu}$ is one-to-one and the extreme points of $H S(p, p)_{R}$ are precisely the kernel functions in equation (2.3.1).

Proof. For each $x \in X$ the function $z^{p} /(1-x z)^{p}(1-\bar{x} z)^{p}$ is in $S(p, p)_{R}$ and, therefore, also in $T(p)$ [7]. Hence $F \subset T(p)$. We next prove that $T(p) \subset F$. If $f(z) \in T^{*}(p)$ we find by repeated applications of Theorem (1) in [7] that 


$$
g(z)=\prod_{k=1}^{p-1} \frac{\left(1-x_{k} z\right)\left(1-\bar{x}_{k} z\right)}{z} f(z) \in T^{*}(1),
$$

where $\left|x_{k}\right|=1, k=1,2, \ldots, p-1$. In [21] it was shown that $T^{*}(1) \subset T$, the class of typically real functions, and so by Theorem (4) in [3] and equation (2.3.2) we have

$$
F(z)=\prod_{k=1}^{p-1} \frac{z}{\left(1-x_{k} z\right)\left(1-\bar{x}_{k} z\right)} \int_{X} \frac{z}{(1-x z)(1-\bar{x} z)} d \mu(x),
$$

$\left|x_{k}\right|=1$ for $k=1,2, \ldots(p-1)$.

Since each factor is typically real, appeal again to Theorem (4) in [3], and an application of Lemma (2.2) shows that $f(z) \in F$.

Now suppose that $f(z) \in T(p)$. Then $f(r z) / r^{p} \in T^{*}(p)$ for all $r$ sufficiently close to 1 . Suppose $r_{n}$ is a sequence of numbers increasing to 1 . By the previous remark and the argument given above we have

$$
\frac{f\left(r_{n} z\right)}{r_{n}^{p}}=\int_{X} \frac{z^{p}}{(1-x z)^{p}(1-\bar{x} z)^{p}} d \mu_{n}(x) .
$$

By the Helly selection principle there exist a subsequence $n_{K}$ and a measure $\mu$ so that

$$
\begin{aligned}
f(z) & =\lim _{k \rightarrow \infty} \int_{X} \frac{z^{p}}{(1-x z)^{p}(1-\bar{x} z)^{p}} d \mu_{n_{k}}(x) \\
& =\int_{X} \frac{z^{p}}{(1-x z)^{p}(1-\bar{x} z)^{p}} d \mu(x) .
\end{aligned}
$$

So we have shown that $T(p)=F$. It is clear from earlier remarks that $F \subset$ $H S(p, p)_{R}$. Suppose that $f(z) \in S(p, p)_{R}$. We know that $f(z)=g(z)^{p}$ where $g(z)$ is starlike univalent and has real coefficients. Hence by a result in [3] we have

$$
f(z)=\left[\int_{X} \frac{z}{(1-x z)(1-\bar{x} z)} d v(x)\right]^{p} .
$$

It follows by Lemma (2.2) that $f(z) \in F$. Hence $T(p)=F=H S(p, p)_{R}$.

Suppose that $p_{n}(x, \bar{x})=x^{n}+\bar{x}^{n}$. In [3] it was proven, in effect, that if

$$
\int_{X} p_{n}(x, \bar{x}) d \mu_{1}=\int_{X} p_{n}(x, \bar{x}) d \mu_{2} \text { for all } n=1,2, \ldots,
$$

then $\mu_{1}=\mu_{2}$. Suppose that we have $f_{\mu_{1}}=f_{\mu_{2}}$. Then we find

$$
\int_{X} \frac{1}{(1-x z)^{p}(1-\bar{x} z)^{p}} d \mu_{1}=\int_{X} \frac{1}{(1-x z)^{p}(1-\bar{x} z)^{p}} d \mu_{2} .
$$


It follows from equation (2.3.8) that equation (2.3.7) holds and hence $\mu_{1}=\mu_{2}$. Since the map $\mu \rightarrow f_{\mu}$ is one-to-one the conclusion about the extreme points follows from Theorem (1) in [3].

REMARK. The first author has obtained [9] the extreme points of the closed convex hull of the $n$-fold symmetric starlike univalent functions with real coefficients. Using this result we can treat the case of functions in $S(p, p)_{R}$ with $\boldsymbol{n}$-fold symmetry. We again omit the details. We note that the case of $\boldsymbol{n}$-fold symmetric typically real functions remains open having only been treated [9] in the case of odd functions.

THEOREM (2.4). $H K(p, p)_{R}=H S(p, p)_{R}=T(p)$ and $\operatorname{EHK}(p, p)_{R}=$ $E H S(p, p)_{R}$.

Proof. We need only prove the first equality, the second being the content of Theorem (2.3) above. Since $S(p, p)_{R} \subset K(p, p)_{R}$ and the kernel functions in equation (2.3.1) are in $S(p, p)_{R}$, it is clear that $T(p) \subset H K(p, p)_{R}$. We show that $K(p, p)_{R} \subset T(p)$.

Suppose $f(z) \in K(p, p)_{R}$. Let $g(z)=z\left(f(z) / z^{p}\right)^{1 / p}=f(z)^{1 / p}$. We then have

$$
1+\frac{z f^{\prime \prime}(z)}{f^{\prime}(z)}=1+\frac{z g^{\prime \prime}(z)}{g^{\prime}(z)}+(p-1) \frac{z g^{\prime}(z)}{g(z)} \text {. }
$$

Since $f(z) \in K(p, p)$ we have [16] that

$$
\int_{\theta_{1}}^{\theta_{2}} \operatorname{Re}\left(1+\frac{z f^{\prime \prime}(z)}{f^{\prime}(z)}\right) d \theta>-\pi \text { for } \theta_{1}<\theta_{2},
$$

and hence by equation (2.4.1), $g(z)$ is Bazilevic of order $p$ [22] and therefore one-to-one. Clearly $g(z)$ has real coefficients. We have, therefore, $f(z)=g(z)^{p}$ where $g(z)$ is typically real. So by Theorem (4) in [3] we have

$$
f(z)=\left[\int_{X} \frac{z}{(1-x z)(1-\bar{x} z)} d v(x)\right]^{p} .
$$

The result follows by direct application of Lemma (2.2). Hence $H K(p, p)_{R}=$ $H S(p, p)_{R}$. The conclusion about the extreme points follows from Theorem (2.3).

REMARK. When $p=1$, the first equation of Theorem (2.4) was proven in [10] while the second was proven in [3].

THEOREM (2.5). Let $X=\{x|| x \mid=1, \operatorname{Im} x \geqslant 0\}, P$ the set of probability measures on $X$, and $F$ the set of functions $f_{\mu}$ on $\Delta$ defined by

$$
f_{\mu}(z)=\int_{X}\left[\int_{0}^{z} \frac{p \tau^{p-1}}{(1-x \tau)^{p}(1-\bar{x} \tau)^{p}} d \tau\right] d \mu(x), \quad \mu \in P .
$$


Then $F=H C(p, p)_{R}$, the map $\mu \rightarrow f_{\mu}$ is one-to-one and the extreme points of $H C(p, p)_{R}$ are precisely the kernel functions in equation (2.5.1).

Proof. Let $L$ denote the operator defined in the proof of Theorem (2.2). It is clear that $L\left(S(p, p)_{R}\right)=C(p, p)_{R}$, and since the operator is a homeomorphism the result follows immediately from Theorem (2.3) or (2.4).

REMARK. We can also treat completely as in Theorem (2.3) the case of $n$-fold symmetric functions in $C(p, p)_{R}$.

THEOREM (2.6). Let $X$ be the torus $\{(x, y):|x|=|y|=1\}$, P the set of probability' measures on $X$,

$$
K(z, x, y)=\int_{0}^{z} p \frac{\tau^{p-1}(1-y \tau)}{(1-x \tau)^{2 p+1}} d \tau
$$

where $z \in \Delta,|x|=|y|=1$, and $x \neq y$ and let $F$ be the set of functions $f_{\mu}$ on $\Delta$ defined by

$$
f_{\mu}(z)=\int_{X} K(z, x, y) d \mu(x, y) \quad \text { where } \mu \in P .
$$

Then $F=H K(p, p)$, the map $\mu \rightarrow f_{\mu}$ is one-to-one, and the extreme points of $H K(p, p)$ are given by the functions in equation (2.6) where $x \neq y$.

Proof. The proof of this theorem follows from Lemma (1.1) with much the same type of argument used to prove this result in the case $p=1$ in [3]. The argument to show that the map $\mu \rightarrow f_{\mu}$ is one-to-one also follows by much the same type of argument made in the case $p=1$. We omit these details.

REMARK. We note that the set of extreme points for $H C(p, p)$ and $H S(p, p)$ is closed. The set of extreme points of $H K(p, p)$ is not closed since the functions in equation (2.6) associated with the case $x=y$ are not in $\operatorname{EHK}(p, p)$. They are actually the extreme points of $H C(p, p)$.

LEMma (2.3). If $x \neq y$, then

$$
K(z, x, y)=\int_{0}^{z} p \frac{p^{p-1}(1-y \tau)}{(1-x \tau)^{2 p+1}} d \tau=\left[\frac{z}{(1-x z)^{2}}\right]^{p} \phi(z)
$$

where

$$
\phi(z)=1+b_{1} z+b_{2} z^{2}+\cdots+b_{p} z^{p}
$$

and

$$
b_{n}=(-1)^{n} \frac{p(p-1) \cdots(p-n+1)}{(p+1)(p+2) \cdots(p+n)} x^{n-1}(x+y) \text { for } 1 \leqslant n \leqslant p
$$


Proof. Let $K(z, x, y)=\left[z /(1-x z)^{2}\right]^{p} \phi(z)$. Then differentiating and simplifying we have

$$
p(1-y z)=(1-x z) z \phi^{\prime}(z)+p(1+x z) \phi(z) .
$$

We let $\phi(z)=1+\sum_{n=1}^{\infty} b_{n} z^{n}$ and substitute in the preceding equation. Comparing coefficients we find inductively that

$$
b_{n}=(-1)^{n} \frac{p(p-1) \cdots(p-n+1)}{(p+1)(p+2) \cdots(p+n)} x^{n-1}(x+y)
$$

for $n=1,2, \ldots, p$ and $b_{n}=0$ if $n \geqslant p+1$.

III. Coefficient estimates for functions majorized by or subordinate to functions in $S(p, p), C(p, p), K(p, p)$ and $T(p)$ and their derivatives. In this section we take advantage of the results of the previous section to solve extremal problems. We remark that these results are well known in the case $p=1$.

THEOREM (3.1). Let $f(z)=\Sigma_{n=0}^{\infty} a_{p+n} z^{p+n}$ be analytic in $\Delta$ and be majorized by some function in $\mathrm{HC}(p, p)$. Then

$$
f(z)<<F(z)=\int_{0}^{z} \frac{p \tau^{p-1}}{(1-\tau)^{2 p}} d \tau .
$$

The result is sharp.

ProOF. Because of results proven in [18] it suffices to assume that $f(z)$ is majorized by some function in $\operatorname{EHC}(p, p)$. We have

$$
f(z)=\phi(z) F(x z) / x^{p},
$$

$|x|=1$ and $\phi$ is analytic in $\Delta$ with $|\phi(z)| \leqslant 1$. We may assume without loss of generality that $x=1$. By Remark (2) made after Theorem (2.2) we see that

$$
f(z)=\phi(z) \frac{z}{(1-z)^{2}} z\left[\frac{z}{(1-z)^{2}}\right]^{p-2} g(z)
$$

where

$$
g(z)=\left[1+\frac{(-1)^{2 p+1} p !\left(\begin{array}{c}
2 p-1 \\
p+1
\end{array}\right)}{(2 p-1) \cdots(p)} z+\cdots+\frac{(-1)^{3 p-1} p !\left(\begin{array}{c}
2 p-1 \\
2 p-1
\end{array}\right)}{(2 p-1) \cdots(p)} z^{p-1}\right] /(1-z) .
$$

It is known [17] that $\phi(z) z /(1-z)^{2}<<z /(1-z)^{2}$ and this is our result for $p=$ 1 , so we may assume here that $p \geqslant 2$. Suppose $g(z)=1+b_{1} z+b_{2} z^{2}+\cdots+$ $b_{n} z^{n}+\cdots$. If we can show that the coefficients $b_{n}$ are real and positive for all $n$ we shall be done. It is easy to see that $b_{n}=b_{p-1}$ for all $n \geqslant p-1$. We note first that

$$
b_{1}=1+(-1)^{2 p+1} \frac{p !\left(\begin{array}{c}
2 p-1 \\
p+1
\end{array}\right)}{(2 p-1) \cdots(p)}=1-\frac{p-1}{p+1}>0
$$


In general,

$$
b_{n}=1+(-1)^{2 p+1} \frac{p !\left(\begin{array}{c}
2 p-1 \\
p+1
\end{array}\right)}{(2 p-1) \cdots(p)}+\cdots+(-1)^{2 p+n} \frac{p !\left(\begin{array}{l}
2 p-1 \\
p+n
\end{array}\right)}{(2 p-1) \cdots(p)},
$$

where $n=1,2, \ldots, p-1$. Since we have alternating signs in the expression for $b_{n}$ and $b_{1}>0$ we need only prove the inequality

$$
\frac{p !\left(\begin{array}{c}
2 p-1 \\
p+m
\end{array}\right)}{(2 p-1) \cdots(p)}>\frac{p !\left(\begin{array}{c}
2 p-1 \\
p+m+1
\end{array}\right)}{(2 p-1) \cdots(p)} \text { for } m=1,2, \ldots, p-1 \text {. }
$$

The previous inequality is clearly true since

$$
\left(\begin{array}{c}
2 p-1 \\
p+m
\end{array}\right)>\left(\begin{array}{c}
2 p-1 \\
p+m+1
\end{array}\right)
$$

Therefore we conclude $b_{n}>0$ for all $n$ and the theorem is proven. The sharpness is obvious.

THEOREM (3.2). Let $f(z)=\sum_{n=0}^{\infty} a_{p+n} z^{p+n}$ be analytic in $\Delta$ and be majorized by some function in $H K(p, p)$. Then

The result is sharp.

$$
f(z)<K K(z)=\left[z /(1-z)^{2}\right]^{p}
$$

Proof. We may assume that $f(z)$ is majorized by some function in $E H K(p, p)$. Hence, by Lemma (2.3) and assuming, without loss of generality, that $x=1$, we have

$$
f(z)=\phi(z) \frac{z^{p}}{(1-z)^{2 p}}\left(1+b_{1}(a) z+\cdots+b_{p}(a) z^{p}\right)
$$

where

$$
b_{n}(a)=(-1)^{n} \frac{p(p-1) \cdots(p-n+1)}{(p+1)(p+2) \cdots(p+n)}(1+a) \text { and }|a|=1
$$

We write equation (3.2.1) as

$$
f(z)=\phi(z) \frac{z}{(1-z)^{2}} \frac{z^{p-1}}{(1-z)^{2 p-3}} \frac{1+b_{1}(a) z+\cdots+b_{p}(a) z^{p}}{1-z}
$$

As mentioned in the proof of Theorem (3.1) we know [17] that $\phi(z) z /(1-z)^{2}$ $\ll z /(1-z)^{2}$. The theorem for $p=2,3, \ldots$ would follow from this remark and equation (3.2.2) if we could prove that

$$
g(z)=\left(1+b_{1}(a) z+\cdots+b_{p}(a) z^{p}\right) /(1-z)<1 /(1-z) .
$$


We recall that the theorem is known for $p=1$ [18]. Let $g(z)=1+c_{1}(a) z+$ $c_{2}(a) z^{2}+\cdots$. It is easy to show that $c_{n}(a)=1+\sum_{k=1}^{n} b_{k}(a)$ and $c_{n}=c_{p}$ if $n \geqslant p$. We claim that $c_{n}(a)=A_{n}+B_{n} a$ where $A_{n}>0$ and $B_{n}<0$. In fact it is clear that

$$
A_{n}=1-\frac{p}{p+1}+\frac{p(p-1)}{(p+1)(p+2)}-\cdots+(-1)^{n} \frac{p(p-1) \cdots(p-n+1)}{(p+1)(p+2) \cdots(p+n)}
$$

and

$$
B_{n}=\frac{-p}{p+1}+\frac{p(p-1)}{(p+1)(p+2)}+\cdots+(-1)^{n} \frac{p(p-1) \cdots(p-n+1)}{(p+1)(p+2) \cdots(p+n)}
$$

We see that $A_{1}>0$ and $B_{1}<0$. Because of the alternating signs we can conclude $A_{n}>0$ and $B_{n}<0$ for all $n$ if the following inequality is valid:

$$
\frac{p(p-1) \cdots(p-k+1)}{(p+1)(p+2) \cdots(p+k)}>\frac{p(p-1) \cdots(p-k+1)(p-k)}{(p+1)(p+2) \cdots(p+k)(p+k+1)}
$$

for fixed $k$ satisfying $1 \leqslant k \leqslant n$. This inequality is clearly true since $(p-k) /(p+k+1)<1$. We conclude that $\left|c_{n}(a)\right| \leqslant c_{n}(-1)=1$ and so $g(z)$ $\ll 1 /(1-z)$. The theorem now follows. The sharpness follows since $K(z)$ $\in K(p, p)$.

REMARK. Since $T(p) \subset H S(p, p) \subset H(K(p, p)$, Theorem (3.2) is also valid for $S(p, p)$ and $T(p)$ for all $p$.

THEOREM (3.3). Let $f(z)=\sum_{n=0}^{\infty} a_{p+n} z^{p+n}$ be analytic in $\Delta$ and be subordinate to some function in $\mathrm{HC}(p, p)$. Then

$$
f(z) \ll F(z)=\int_{0}^{z} \frac{p \tau^{p-1}}{(1-\tau)^{2 p}} d \tau .
$$

The result is sharp.

Proof. The arguments given in [18] show that in order to maximize $\left|a_{n}\right|$ we need only consider the functions $f$ which are subordinate to a function in $\operatorname{EHC}(p, p)$. Therefore by Theorem (2.2) we have

$$
f(z)=\int_{0}^{\phi(z)} \frac{p \tau^{p-1}}{(1-x \tau)^{2 p}} d \tau
$$

where $|x|=1, \phi(z)$ is analytic for $|z|<1,|\phi(z)|<1$, and $\phi(0)=0$. Without loss of generality we may assume $x=1$. Hence

$$
f^{\prime}(z)=\left[\frac{\phi(z)}{(1-\phi(z))^{2 !}}\right]^{p-1} p \frac{\phi^{\prime}(z)}{(1-\phi(z))^{2}}
$$


It is known [4] that $\phi(z) /(1-\phi(z))^{2}<<z /(1-z)^{2}$ and so we conclude

$$
\left[\frac{\phi(z)}{(1-\phi(z))^{2}}\right]^{p-1} \ll\left[\frac{z}{(1-z)^{2}}\right]^{p-1} \text {. }
$$

It is also known that $1 /(1-\phi(z))<<1 /(1-z)[11]$. Hence

$$
\phi^{\prime}(z) /(1-\phi(z))^{2}=[1 /(1-\phi(z))]^{\prime} \ll 1 /(1-z)^{2} .
$$

It now follows that $f^{\prime} \ll F^{\prime}$ and the theorem is proven. The sharpness follows since $F(z) \in C(p, p)$.

THEOREM (3.4). Let $f(z)=\Sigma_{n=0}^{\infty} a_{p+n} z^{p+n}$ be analytic in $\Delta$ and be subordinate to some function in $H K(p, p)$. Then

$$
f(z) \ll\left[z /(1-z)^{2}\right]^{p}=K(z) .
$$

The result is sharp.

Proof. We may assume that $f(z)$ is subordinate to a function in $E H K(p, p)$. Hence, by Theorem (2.6) we have

$$
f^{\prime}(z)=p \frac{\phi(z)^{p-1}}{(1-x \phi(z))^{2 p+1}}(1-y \phi(z)) \phi^{\prime}(z)
$$

where $|x|=|y|=1$ and $\phi(0)=0,|\phi(z)|<1$ and $\phi$ is analytic in $\Delta$. Without loss of generality assume $x=1$. Then we have

$$
f^{\prime}(z)=\left[\frac{\phi(z)}{1-\phi(z)}\right]^{p-1} p \frac{\phi^{\prime}(z)}{(1-\phi(z))^{p+1}} \frac{1-y \phi(z)}{1-\phi(z)} .
$$

It is known [11] that $(1-\phi(z))^{-p} \ll(1-z)^{-p}$ for $p=1,2,3, \ldots$.

Since $p \phi^{\prime}(z) /(1-\phi(z))^{p+1}=\left[1 /(1-\phi(z))^{p}\right]^{\prime}$ we conclude that

$$
p \phi^{\prime}(z) /(1-\phi(z))^{p+1} \ll p /(1-z)^{p+1} \text {. }
$$

It is well known that

$$
\frac{\phi(z)}{1-\phi(z)} \ll \frac{z}{1-z} \text { and } \frac{1-y \phi(z)}{1-\phi(z)} \ll \frac{1+z}{1-z} .
$$

So equation (3.4.1) gives

$$
f^{\prime}(z) \ll\left(\frac{z}{1-z}\right)^{p-1} p \frac{1}{(1-z)^{p+1}} \frac{1+z}{1-z}=K^{\prime}(z)
$$

and the result follows. The sharpness follows since $K(z) \in S(p, p) \subset K(p, p)$.

Remarks. Since $T(p) \subset H S(p, p) \subset H K(p, p)$, Theorem (3.4) also applies to the classes $T(p)$ and $S(p, p)$. 
Our next four theorems appear to be unknown even in the case $p=1$. For any class of analytic functions $A$ we let $A^{\prime}$ denote the class of derivatives.

THEOREM (3.5). $f^{\prime}(z)=\left(\sum_{n=0}^{\infty} a_{p+n} z^{p+n}\right)^{\prime}$ be analytic in $\Delta$ and be majorized by some function in $H C^{\prime}(p, p)$. Then

$$
f^{\prime}(z) \ll F^{\prime}(z)=p z^{p-1} /(1-z)^{2 p} .
$$

The result is sharp.

Proof. We have, without loss of generality, as in the proof of Theorem (3.1),

$$
f^{\prime}(z)=\phi(z) p \frac{z^{p-1}}{(1-z)^{2 p}}=\phi(z) \frac{z}{(1-z)^{2}} p \frac{z^{p-2}}{(1-z)^{2 p-2}}
$$

where $|\phi(z)|<1$ and $\phi(z)$ is analytic in $\Delta$. Since $\phi(z) z /(1-z)^{2}<<z /(1-z)^{2}$ [17], the theorem follows.

ThEOREM (3.6). Let $f^{\prime}(z)=\left(\sum_{n=0}^{\infty} a_{p+n} z^{p+n}\right)^{\prime}$ be analytic in $\Delta$ and be majorized by some function in $H K^{\prime}(p, p)$. Then

$$
f^{\prime}(z) \ll K^{\prime}(z)=\left[z^{p} /(1-z)^{2 p}\right]^{\prime} .
$$

The result is sharp.

Proof. We have without loss of generality, for $|a|=1$,

$$
\begin{aligned}
f^{\prime}(z) & =\phi(z) p \frac{z^{p-1}}{(1-z)^{2 p+1}}(1-a z) \\
& =\phi(z) \frac{z}{(1-z)^{2}} p \frac{z^{p-2}}{(1-z)^{2 p-2}} \frac{1+a z}{1-z} .
\end{aligned}
$$

Since

$$
\phi(z) \frac{z}{(1-z)^{2}}<<\frac{z}{(1-z)^{2}} \text { and } \frac{1+a z}{1-z}<\frac{1+z}{1-z}
$$

the theorem follows directly from equation (3.6.1).

REMARK. Since $T^{\prime}(p) \subset H S^{\prime}(p, p) \subset H K^{\prime}(p, p)$, Theorem (3.6) also holds for $T^{\prime}(p)$ and $S^{\prime}(p, p)$.

THEOREM (3.7). Let $f^{\prime}(z)=\left(\sum_{n=0}^{\infty} a_{p+n} z^{p+n}\right)^{\prime}$ be analytic in $\Delta$ and be subordinate to some function in $H C^{\prime}(p, p)$. Then

$$
f^{\prime}(z) \ll F^{\prime}(z)=p z^{p-1} /(1-z)^{2 p} \text {. }
$$

The result is sharp.

Proof. We may assume without loss of generality for familiar reasons that 


$$
f^{\prime}(z)=\frac{p \phi(z)^{p-1}}{(1-\phi(z))^{2 p}}=\left[\frac{\phi(z)}{(1-\phi(z))^{2}}\right]^{p-1} \frac{p}{(1-\phi(z))^{2}}
$$

The theorem follows from equation (3.7.1) by now familiar arguments.

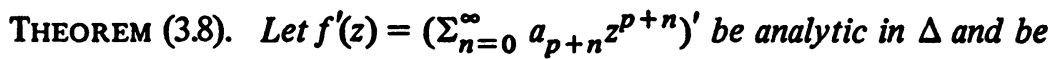
subordinate to some function in $H K^{\prime}(p, p)$. Then

$$
f^{\prime}(z) \ll K^{\prime}(z)=\left[z^{p} /(1-z)^{2 p}\right]^{\prime} .
$$

The result is sharp.

Proof. We may assume that, for $|a|=1$,

$$
\begin{aligned}
f^{\prime}(z) & =p \frac{\phi(z)^{p-1}}{(1-\phi(z))^{2 p+1}}(1-a \phi(z)) \\
& =\left(\frac{\phi(z)}{1-\phi(z)}\right)^{p-1} p \frac{1}{(1-\phi(z))^{p+1}} \frac{1-a \phi(z)}{1-\phi(z)} .
\end{aligned}
$$

The result now follows from equation (3.8.2) by familiar arguments.

REMARK. Since $T^{\prime}(p) \subset H S^{\prime}(p, p) \subset H K^{\prime}(p, p)$, Theorem (3.8) also holds for $T^{\prime}(p)$ and $S^{\prime}(p, p)$.

IV. Estimates on $L^{q}$ means of multivalent functions. In this section we seek to maximize the subadditive continuous functional defined by

$$
J\left(f^{(n)}\right)=\int_{0}^{2 \pi}\left|f^{(n)}\left(r e^{i \theta}\right)\right|^{q} d \theta, \quad n=0,1,2, \ldots,
$$

and $f$ belongs to one of the families of multivalent functions which we studied in §II. As pointed out in [18] for $q \geqslant 1$ we have

$$
\max _{f \in F} J\left(f^{(n)}\right)=\max _{f \in E H F} J\left(f^{(n)}\right)
$$

for any compact family $F$.

In the case $0<q<1$ and $n=0$ we are able, using results in $\S \mathrm{V}$, to treat the classes $C(p, p)$ for $p=2,3$ and $S(p, p)$ for all $p$. For these problems, i.e., maximizing $J(f)$, the main tools are a subordination result and Littlewood's Theorem [15], which says that if $f<g$ then $J(f) \leqslant J(g)$ for all $q>0$ and $n=0$.

THEOREM (4.1). If $f(z) \in H C(p, p), q \geqslant 1, n=0,1,2, \ldots$, then

$$
J\left(f^{(n)}\right) \leqslant J\left(F^{(n)}\right) \text { where } F(z)=\int_{0}^{z} \frac{p \tau^{p-1}}{(1-\tau)^{2 p}} d \tau .
$$

Proof. As mentioned above it suffices to prove the result when $f(z) \in$ $E H C(p, p)$. But it is easily seen that the functional $J\left(f^{(n)}\right)$ is constant on the set of extreme points as determined in Theorem (2.2) and, of course, $F(z) \in$ $E H C(p, p)$ corresponding to the choice $x=1$. 
REMARKS. (1) This result clearly includes the class $C(p, p)_{R}$ since $F(z)$ $\in C(p, p)_{R}$.

(2) If $n=0, p=2$ or 3 and $0<q<1$, then the same conclusion follows from Theorem (5.1) in $\S \mathrm{V}$ and Littlewood's inequality mentioned above [15].

TheOREM (4.2). If $f(z) \in H S(p, p), q \geqslant 1, n=0,1,2, \ldots$, then

$$
J\left(f^{(n)}\right) \leqslant J\left(K^{(n)}\right) \text { where } K(z)=z^{p} /(1-z)^{2 p} .
$$

Proof. By considering the set $\operatorname{EHS}(p, p)$ as determined in Theorem (2.1) we find that the functional $J\left(f^{(n)}\right)$ is constant on the set of extreme points and also that $K(z) \in E H S(p, p)$. The result follows.

REMARKS. We claim that if $f(z) \in S(p, p)$, then for $p=1,2, \ldots$, $f(z) / z^{p}<K(z) / z^{p}$ where $K(z)$ is given in Theorem (4.2). This can easily be proven from the known result of Strohhicker [23] in the case $p=1$ and the fact that each function in $S(p, p)$ is the $p$ th power of a function in $S(1,1)$. From this subordination and Littlewood's inequality [15] we conclude $J(f) \leqslant$ $J(K)$ for $0<q<1$ and arbitrary $p$.

We also note that the conclusion of Theorem (4.2) also holds for the class $T(p)$ since, as we have observed elsewhere, $T(p) \subset H S(p, p)$. We recall that each of the kernel functions in equation (2.3.1) is in $S(p, p)$.

THEOREM (4.3). If $f(z) \in H K(p, p), q=1,2, \ldots$ and $n=0$, then

$$
J(f) \leqslant J(K) \text { where } K(z)=z^{p} /(1-z)^{2 p} .
$$

Proof. It suffices to assume that $f(z) \in \operatorname{EHK}(p, p)$ as determined in Theorem (2.6).

We may suppose also that the parameter $x$ is chosen to be 1 . Hence, by Lemma (2.3) where $x=1$, we find

$$
\int_{0}^{2 \pi}\left|\frac{f(z)}{z^{p}}\right|^{q} d \theta=\int_{0}^{2 \pi}\left|\frac{1}{(1-z)^{2 p}} \phi(z)\right|^{q} d \theta
$$

where $\phi(z)=1+b_{1} z+\cdots+b_{p} z^{p}$ and the numbers $b_{1}, b_{2}, \ldots, b_{p}$ are given explicitly in Lemma (2.3). By applying the Cauchy-Schwarz inequality to the right-hand side of equation (4.3.1) we find

$$
\begin{aligned}
\int_{0}^{2 \pi}\left|\frac{f(z)}{z^{p}}\right|^{q} d \theta \leqslant & \left(\int_{0}^{2 \pi}\left|\left[\frac{1+b_{1} z+\cdots+b_{p} z^{p}}{(1-z)^{p}}\right]^{q}\right|^{2} d \theta\right)^{1 / 2} \\
& \times\left(\int_{0}^{2 \pi} \frac{1}{|1-z|^{2 p q}} d \theta\right)^{1 / 2} .
\end{aligned}
$$


We claim that

$$
\int_{0}^{2 \pi}\left|\left[\frac{1+b_{1} z+\cdots+b_{p} z^{p}}{(1-z)^{p}}\right]^{q}\right|^{2} d \theta \leqslant \int_{0}^{2 \pi} \frac{1}{|1-z|^{2 p g}} d \theta
$$

from which the result follows. In the proof of Theorem (3.2) we showed that

$$
\left(1+b_{1} z+\cdots+b_{p} z^{p}\right) /(1-z)<1 /(1-z) .
$$

Equation (4.3.4) implies that

$$
\left(1+b_{1} z+\cdots+b_{p} z^{p}\right) /(1-z)^{p}<1 /(1-z)^{p} .
$$

Our claim now follows by familiar arguments [18] which utilize Parseval's identity.

REMarks (1) Using the methods of D. R. Wilken [26] we can show that Theorem (4.3) would also hold for all $q \geqslant 1$ if the following conjecture were true.

Conjecture. $\left(1+b_{1} z+\cdots+b_{p} z^{p}\right)^{s} /(1-z)<1 /(1-z)$ where $1 / 2 \leqslant S \leqslant 1$. We have been unable to prove this except in the case $S=1$.

(2) Theorem (2.6) implies that if $f(z) \in K(p, p)$ then $f(z) \ll K(z)$. See also Umezawa [25]. Hence it follows that $f^{(n)}(z) \ll K^{(n)}(z)$ for all $n=0,1$, $2, \ldots$. Using this fact and the technique [18] utilizing Parseval's identity it is easy to prove that

$$
J\left(f^{(n)}\right) \leqslant J\left(K^{(n)}\right) \text { for all } n=0,1,2, \ldots
$$

whenever $q=2,4,6, \ldots$, i.e., is an even integer.

(3) We have not touched the question of whether equality occurs only for $f=K$.

THEOREM (4.4). If $f(z) \in H K(p, p), q \geqslant 1$, then

$$
J\left(f^{(1)}\right) \leqslant J\left(K^{(1)}\right) \text { where } K(z)=z^{p} /(1-z)^{2 p} .
$$

Proof. It suffices to assume that $f(z) \in E H K(p, p)$. We may also suppose without loss of generality that $x=1$. We wish to prove

$$
\int_{0}^{2 \pi}\left|\frac{1-a z}{(1-z)^{2 p+1}}\right|^{q} d \theta \leqslant \int_{0}^{2 \pi}\left|\frac{1+z}{(1-z)^{2 p+1}}\right|^{q} d \theta \quad \text { where }|a|=1
$$

The symmetric decreasing rearrangement (as defined in [8]) of $\left|(1-z)^{2 p+1}\right|^{-q}$ is itself and the rearrangement of $|1-a z|^{q}$ is $|1+z|^{q}$ and so equation (4.4) is an immediate consequence of a result in [5].

REMARK. We have been unable to make progress on this problem when $n \geqslant 2$ and $q$ is not an even integer. 
V. A subordination theorem for $C(p, p)$. In this section we employ a method recently used by MacGregor [19] to obtain subordination theorems for $z f^{\prime}(z) / f(z)$ and $f(z) / z^{p}$ when $f$ is in $C(p, p)$ and $p=2$ and 3. The key to the proof lies in a recent lemma proven by I. S. Jack [13] and the fact that certain functions are univalent. This is the subject of the next three lemmas.

LEMMA (5.1) [I. S. JACK]. Let $w(z)$ be analytic for $|z| \leqslant r$ with $w(0)=0$. Suppose $z_{1},\left|z_{1}\right|=r$, is such that $\left|w\left(z_{1}\right)\right|=\max _{|z|=r}|w(z)|$; then $z_{1} w^{\prime}\left(z_{1}\right)=$ $k w\left(z_{1}\right)$ where $k \geqslant 1$.

LEMMA (5.2). Let

$$
F(z)=p \int_{0}^{z} \frac{\tau^{p-1}}{(1-\tau)^{2 p}} d \tau=\frac{\Sigma_{m=p}^{2 p-1}(-1)^{m+p} p !\left(\begin{array}{c}
2 p-1 \\
m
\end{array}\right) z^{m}}{(2 p-1) \cdots(p)(1-z)^{2 p-1}}
$$

then for $p=2,3, z F^{\prime}(z) / F(z)-p$ is univalent in $\Delta$ and maps $\Delta$ onto a domain starlike with respect to the origin. In particular then, $z F^{\prime}(z) / F(z)$ is univalent in $\Delta$

Proof. When $p=2$, let $G(z)=z F^{\prime}(z) / F(z)-2=\left(8 z-2 z^{2}\right) /\left(3-4 z+z^{2}\right)$. We need to prove that $\operatorname{Re} z G^{\prime}(z) / G(z)>0$ for $z \in \Delta$. Letting $z=e^{i \theta}$ and $x=$ $\cos \theta$ it can be seen that

$$
\frac{z G^{\prime}(z)}{G(z)}=\frac{6(1-x)\left(13-13 x+4 x^{2}\right)}{(1-x)\left|12-7 e^{i \theta}+e^{2 i \theta}\right|^{2}}
$$

Thus we need only show that $p(x)=13-13 x+4 x^{2}$ is positive for $-1 \leqslant x \leqslant$ 1. This is obvious.

When $p=3$, let

$$
G(z)=\frac{z F^{\prime}(z)}{F(z)}-3=\frac{45 z-18 z^{2}+3 z^{3}}{10-15 z+6 z^{2}-z^{3}},
$$

then

$$
\frac{z G^{\prime}(z)}{G(z)}=\frac{10\left(15-12 z+3 z^{2}\right)}{\left(10-15 z+6 z^{2}-z^{3}\right)\left(15-6 z+z^{2}\right)} .
$$

Again we need to prove that $\operatorname{Re} z G^{\prime}(z) / G(z)>0$ for $z$ in $\Delta$. Letting $z=e^{i \theta}$, tedious computations show that we need to prove that the expression $6,240-9,408 \cos \theta+4,128 \cos 2 \theta-1,137 \cos 3 \theta+192 \cos 4 \theta-15 \cos 5 \theta$ is positive for $0 \leqslant \theta \leqslant 2 \pi$. Letting $x=\cos \theta$ in the previous expression and noticing that the resulting polynomial has a factor of $(1-x)$, it is seen that we need to prove that the polynomial $p(x)=96-157 x+123 x^{2}-54 x^{3}+10 x^{4}$ is positive for $-1 \leqslant x \leqslant 1$. $p^{(2)}(x)$ is never zero and thus positive for all $x$. Thus for $-1 \leqslant x \leqslant 1, p^{\prime}(x) \leqslant p^{\prime}(1)<0$. Therefore $p(x) \geqslant p(1)>0$ for $-1 \leqslant$ $x \leqslant 1$. 
Lemma (5.3). Let $F(z)$ be as in Lemma (5.2), $G(z)=z F^{\prime}(z) / F(z), H(z)=$ $1+{ }_{z} F^{\prime \prime}(z) / F^{\prime}(z)$ and $H_{k}(z)=k H(z)+(1-k) G(z), k \geqslant 1$; then for $p=2,3$, $H_{k}(z)$ is univalent in $\Delta$ and $H \prec H_{k}$.

Proof. For any value of $p, H(z)=p(1+z) /(1-z)$ maps $\Delta$ onto a convex domain. Thus it is sufficient to prove that $\operatorname{Re} H_{k}^{\prime}(z) / H^{\prime}(z)>0$ for $z \in \Delta$ [14]. But since $H_{k}^{\prime}(z) / H^{\prime}(z)=k+(1-k) G^{\prime}(z) / h^{\prime}(z)$ and $k \geqslant 1$, it is sufficient to prove that $\operatorname{Re} G^{\prime}(z) / H^{\prime}(z)<1$ for $z \in \Delta$.

When $p=2$, we need to prove that $\operatorname{Re}\left(1-G^{\prime}(z) / H^{\prime}(z)\right)=$ $\operatorname{Re}\left[\left(3-3 z+z^{2}\right) /\left(9-6 z+z^{2}\right)\right]>0$ for $z$ in $\Delta$. Letting $z=e^{i \theta}$, this is seen to be true if the expression $46-54 \cos \theta+12 \cos 2 \theta$ is positive for $0 \leqslant \theta \leqslant 2 \pi$. If $x=\cos \theta$, this is equivalent to proving that the polynomial $p(x)=24 x^{2}-$ $54 x+34$ is positive for $-1 \leqslant x \leqslant 1$. But $p(x)$ is decreasing on $1 \leqslant x \leqslant 1$ and so $p(x) \geqslant p(1)=4>0$.

When $p=3$, we need to show that

$$
\operatorname{Re}\left(1-\frac{G^{\prime}(z)}{H^{\prime}(z)}\right)=\operatorname{Re}\left(\frac{25-40 z+30 z^{2}-10 z^{3}+z^{4}}{\left(10-5 z+z^{2}\right)^{2}}\right)>0
$$

for $z$ in $\Delta$. Going through the same procedure as the previous case, long but straightforward computations reduce the problem to proving that the polynomial $p(x)=619-1,975 x+2,550 x^{2}-1,390 x^{3}+250 x^{4}$ is positive for $-1 \leqslant x$ $\leqslant 1$. It is seen that $p^{(2)}(x)>0$ for $-1 \leqslant x<x_{0}$ and $p^{(2)}(x)<0$ for $x_{0}<x$ $\leqslant 1$ where $x_{0}=(417-\sqrt{20,889}) / 300$. Using the estimates $68 / 75<x_{0}<$ $91 / 100$, it can be determined that $p^{\prime}\left(x_{0}\right)<0$ and thus $p^{\prime}(x)<0$ for $-1 \leqslant x \leqslant$ 1. Thus $p(x) \geqslant p(1)>0$ for $-1 \leqslant x \leqslant 1$.

To prove that $H<H_{k}$ we follow the proof given by MacGregor [19]. What we say holds for either $p=2$ or 3. Since $H(0)=H_{k}(0)=p$ and since $H_{k}(z)$ is univalent we need only prove that $H(\Delta) \subset H_{k}(\Delta)$. The function $H(z)$ maps $\Delta$ onto the right half plane and if $|z|=1, z \neq 1, \operatorname{Re} H(z)=0$. Thus we need only prove that the boundary values of $H_{k}(z)$ satisfy $\operatorname{Re} w \leqslant 0$. Let $\left|z_{1}\right|=1$, $z_{1} \neq 1, w_{1}=\lim _{z \rightarrow z_{1}} H(z)$ and $w_{2}=\lim _{z \rightarrow z_{1}} G(z)$, since $C(p, p) \subset S(p, p)$, $\operatorname{Re} w_{1}=0$ and $\operatorname{Re} w_{2} \geqslant 0$. Thus $\operatorname{Re}\left(k w_{1}+(1-k) w_{2}\right)=(1-k) \operatorname{Re} w_{2} \leqslant 0$ completing the proof of the lemma.

THEOREM (5.1). Let $f(z)$ be in $C(p, p)$ and $F(z)$ be as in Lemma (5.2), then for $p=2,3$,

$$
z f^{\prime}(z) / f(z)<z F^{\prime}(z) / F(z) \text { and } f(z) / z^{p} \prec F(z) / z^{p} \text {. }
$$

Proof. Having proved Lemmas (5.2) and (5.3) the proof that $z f^{\prime}(z) / f(z) \prec z F^{\prime}(z) / F(z)$ follows exactly as in MacGregor [19]. 
The second half of the theorem follows directly from the first half of the theorem, Theorem (2) in [12], and Lemma (5.2).

REMARK. From the proof of the theorem it is obvious that the theorem would be true for all $p=1,2,3, \ldots$, if Lemmas (5.2) and (5.3) could be proven true for $p=1,2,3, \ldots$.

\section{REFERENCES}

1. J. Bender, Some extremal theorems for multivalently starlike functions, Duke Math. J. 29 (1962), 101-106. MR 24 \#A2018.

2. D. A. Brannan, J. G. Clunie and W. E. Kirwan, On the coefficient problem for functions of bounded boundary rotation, Ann. Acad. Sci. Fenn. Ser. AI No. 523 (1973). MR 49 \#3108.

3. L. Brickman, T. H. MacGregor and D. R. Wilken, Convex hulls of some classical families of univalent functions, Trans. Amer. Math. Soc. 156 (1971), 91-107. MR 43 \#494.

4. L. Brickman, D. J. Hallenbeck, T. H. MacGregor and D. R. Wilken, Convex hulls and extreme points of families of starlike and convex mappings, Trans. Amer. Math. Soc. 185 (1973), 413-428 (1974). MR 49 \#3102.

5. J. Clunie and P. L. Duren, Addendum: An arclength problem for close-to-convex functions, J. London Math. Soc. 41 (1966), 181-182. MR 32 \#7725.

6. A. W. Goodman, On the Schwarz-Christoffel transformation and p-valent functions, Trans. Amer. Math. Soc. 68 (1950), 204-223. MR 11, 508.

7. A. W. Goodman and M. S. Robertson, A class of multivalent functions, Trans. Amer. Math. Soc. 70 (1951), 127-136. MR 12, 691.

8. G. H. Hardy, J. E. Littlewood and G. Pólya, Inequalities, 2nd ed., Cambridge Univ. Press, New York, 1952. MR 13, 727.

9. D. J. Hallenbeck, Convex hulls and extreme points of families of starlike and close-to-convex mappings, Pacific J. Math. 57 (1975), 167-176.

10. Convex hulls and extreme points of some families of univalent functions, Trans. Amer. Math. Soc. 192 (1974), 285-292. MR 49 \#3103.

11. D. J. Hallenbeck and T. H. MacGregor, Subordination and extreme point theory, Pacific J. Math. 50 (1974), 455-468.

12. D. J. Hallenbeck and Stephan Ruscheweyh, Subordination by convex functions, Proc. Amer. Math. Soc. 52 (1975), $191-195$.

13. I. S. Jack, Functions starlike and convex of order $\alpha$, J. London Math. Soc. (2) 3 (1971), 469-474. MR 43 \#7611.

14. W. Kaplan, Close-to-convex schlicht functions, Michigan Math. J. 1 (1952), 169185 (1953). MR 14, 966.

15. J. E. Littlewood, On inequalities in the theory of functions, Proc. London Math. Soc. (2) 23 (1925), $481-519$.

16. A. E. Livingston, p-valent close-to-convex functions, Trans. Amer. Math. Soc. 115 (1965), 161-179. MR 33 \#7520.

17. T. H. MacGregor, Majorization by univalent functions, Duke Math. J. 34 (1967), 95-102. MR 34 \#6062.

18. Applications of extreme-point theory to univalent functions, Michigan

Math J. 19 (1972), 361-376. MR 47 \#477.

19. A subordination for convex functions of order $\alpha, J$. London Math. Soc. (2) 9 (1975), 513-517. 40-67.

20. A. Marx, Untersuchungen über schlichte Abbildungen, Math. Ann. 107 (1932/33),

21. M. S. Robertson, Analytic functions starlike in one direction, Amer. J. Math. 58 (1936), 465-472. $135-142$

22. T. Sheil-Small, On Bazilević functions, Quart. J. Math. Oxford Ser. (2) 23 (1972), 
23. E. Strohhäcker, Beiträge zur Theorie der schlichten Funcktionen, Math Z, 37 (1933), 356-380.

24. T. J. Suffridge, Some remarks on convex maps of the unit disk, Duke Math. J. 37 (1970), 775-777. MR 42 \#722.

25. T. Umezawa, Multivalently close-to-convex functions, Proc. Amer. Math. Soc. 8 (1957), 869-874. MR 19, 846.

26. D. R. Wilken, The integral means of close-to-convex functions, Michigan Math. J. 19 (1972), 377-379. MR 47 \#50.

DEPARTMENT OF MATHEMATICS, UNIVERSITY OF DELAWARE, NEWARK, DELAWARE 19711 